\title{
Propriété intellectuelle et régulation des marchés des biens informationnels : le cas du nommage sur l'Internet
}

Philippe Barbet et Isabelle Liotard

\section{(2) OpenEdition \\ Journals}

Édition électronique

URL : https://journals.openedition.org/rei/4147

DOI : 10.4000/rei.4147

ISSN : 1773-0198

Éditeur

De Boeck Supérieur

Édition imprimée

Date de publication : 15 juin 2010

Pagination : 119-138

ISSN : 0154-3229

Référence électronique

Philippe Barbet et Isabelle Liotard, «Propriété intellectuelle et régulation des marchés des biens informationnels : le cas du nommage sur l'Internet », Revue d'économie industrielle [En ligne], 129-130 | 1 er et 2 e trimestres 2010, document 6, mis en ligne le 01 juin 2012, consulté le 02 juin 2022. URL : http://journals.openedition.org/rei/4147 ; DOI : https://doi.org/10.4000/rei.4147 


\section{PROPRIÉTÉ INTELLECTUELLE ET RÉGULATION DES MARCHÉS DES BIENS INFORMATIONNELS : LE CAS DU NOMMAGE SUR L'INTERNET}

Mots-clés : Propriété intellectuelle, Internet, régulation, noms de domaine

Key words : Intellectual Property Rights, Internet, Regulation, Domain Names

$\mathrm{L}$

a propriété intellectuelle est au centre des processus de création de la valeur et de marchandisation des activités liées à l'économie de l'information. La diffusion des biens informationnels, liée en grande partie au développement d'Internet, conduit à rendre la propriété intellectuelle essentielle dans la régulation des marchés. Ainsi, au niveau international, l'accord «ADPIC » (Accord sur les aspects des droits de propriété intellectuelle qui touchent au commerce) est un de fondements de l'OMC (Organisation Mondiale du Commerce). Au-delà de sa fonction de régulation de marchés existants, la propriété intellectuelle peut également être à l'origine de la création de nouveaux marchés. La naissance et la marchandisation d'une ressource technique comme les noms de domaine sur l'Internet conduisant à de multiples stratégies de la part des demandeurs et des offreurs résultent essentiellement de l'assimilation de cette ressource à une marque, qu'il est alors rentable d'acquérir et de protéger.

Pour mieux comprendre les enjeux autour des noms de domaine, il nous semble utile de revenir dans une première partie sur les différents facteurs qui ont conduit au renforcement des droits de propriété intellectuelle (DPI) et de

(1) UFR Sciences économiques et de Gestion - 99 avenue JB Clément - 93430 Villetaneuse France.

barbet.philippe@univ-paris13.fr 
leur usage stratégique par les firmes, depuis environ une vingtaine d'années. Cet environnement favorable vers davantage de propriété intellectuelle cristallise les tensions autour des brevets, du droit d'auteur et des marques. Ce cadre général touche aussi le monde numérique et en particulier l'Internet où des solutions tentent d'être apportées soit pour appliquer des outils juridiques classiques à ces objets informationnels, soit pour faire évoluer le droit en créant de nouveaux outils de protection. En effet, alors qu'un bien « classique » du monde physique peut être protégé par un brevet ou un droit d'auteur sans réelle difficulté, il n'en va pas de même pour les biens informationnels qui, par leurs caractéristiques intrinsèques, posent problème en matière de protection. En effet, un bien informationnel pur a une caractéristique de bien public (Arrow 1962) : l'utilisation qu'un agent fait de ce bien n'empêche aucunement un autre agent de l'utiliser; par ailleurs, de par sa nature, la diffusion de ce bien est difficilement contrôlable (en l'absence de tout droit de propriété). Les biens informationnels répondent alors à ces critères. De plus, un bien diffusé sur Internet est par nature numérique: il est en général coûteux à produire et quasi gratuit à reproduire et à diffuser (Varian et Shapiro, 1999). Dans ces circonstances, il est clair qu'un bien numérique est en proie à des problèmes de protection dès lors qu'il est disponible sur le réseau Internet.

Parmi les biens informationnels, nous nous sommes centrés sur le système du nommage sur l'Internet que nous analyserons dans une seconde partie. Comme la numérotation téléphonique, ce système était à l'origine une opération technique indispensable au fonctionnement du réseau mais n'avait a priori pas vocation à générer un marché. Pourtant, les noms de domaine, notamment de second niveau, font l'objet de transactions et de stratégies de valorisation de plus en plus complexes des offreurs et des demandeurs et ont les attributs des marchés les plus élaborés (existence d'un premier et d'un second marché, naissance et développement très rapide d'une offre de l'enregistrement, comportements spéculatifs des acheteurs et enfin émergence de litiges qu'il faut arbitrer). Nous montrerons que la naissance et le développement de ce marché sont liés à l'assimilation des noms de domaine à des marques commerciales. Cette évolution a eu des conséquences que nous étudierons. Tout particulièrement, nous présenterons l'émergence de l'industrie de l'enregistrement et de la gestion des noms de domaine dont nous montrerons les principales modalités de régulation et la multiplication des conflits, liés à la propriété intellectuelle, conduisant à la mise en place de modalités particulières de règlement.

\section{I. - LES RÉGIMES DE PROPRIÉTÉ INTELLECTUELLE AU CEUR DE LA RÉGULATION DES MARCHÉS DES BIENS INFORMATIONNELS}

Alors que le système de la propriété intellectuelle est relativement ancien, c'est depuis environ 20 ans que l'on assiste à une accélération sans précédent de son ampleur, de son usage, des difficultés qu'elle engendre et des réponses 
apportées (renforcement des droits versus ouverture). Même si au cours de l'histoire économique ces actifs de protection des inventions ou des œuvres ont fait l'objet de stratégies diverses et variées par les acteurs selon les époques (Kline et Rivette, 2000; Coriat et Weinstein, 2008), la période récente atteste d'une amplification et d'une application marquée des titres de propriété intellectuelle à un nombre toujours plus grand d'objets (notamment numériques) cristallisant encore davantage la frénésie des acteurs. Cette tendance s'est accentuée avec l'arrivée de l'économie numérique et d'Internet qui ont mis sur le devant de la scène un certain nombre de questionnements relatifs à la protection des inventions ou des œuvres. Dans une économie tournée vers la connaissance, Internet permet une reproduction et un transfert des biens informationnels à coûts quasi nuls (2). Cette situation conduit les internautes à une boulimie de consommation de tels biens (par exemple le téléchargement musical illégal) mais sans respecter les droits qui y sont attachés (3) car estimant que tout doit être « gratuit ». De plus, le phénomène récent de l'open innovation (Von Hippel, 2005; Chesbrough 2006) pose aussi des questions en matière de propriété intellectuelle. Cette démarche qui ouvre la voie à de nouvelles formes d'organisation de l'innovation tend à faire « sortir » les entreprises de leur laboratoire interne pour favoriser, développer et valoriser leurs innovations d'une autre manière. La plate-forme Innocentive par exemple permet à l'entreprise qui est face à un problème d'innovation de « poster » des « défis », auxquels vont répondre les chercheurs internautes du monde entier, rétribués par des primes. Là encore, les questions de propriété intellectuelle sont au centre de ces relations touchant le crowdsourcing (4) et conduisent à de nouvelles formes de gestion (via notamment des clauses spécifiques signées entre les protagonistes).

(2) La littérature économique souligne en effet que ces biens informationnels sont élaborés avec des coûts de production élevés (coûts liés à l'investissement proprement dit mais aussi coûts liés au marketing, à la publicité qui s'avèrent irrécupérables en cas d'échec) alors que les coûts de diffusion se réduisent avec Internet (la copie numérique se fait instantanément et à coût quasi nul). Toutefois, le téléchargement illégal de l'internaute risque de se voir alourdir par les nouvelles mesures de la loi Hadopi 2 en France, au travers desquelles des sanctions (pécuniaires) sont envisagées.

(3) Cette situation conduit notamment la France à s'appuyer sur le volet législatif pour tenter de résoudre le problème. Après l'échec de la solution dite de « licence globale », et la loi DADVSI de juin 2006 (Droit d'Auteur Droits Voisins appliqués à la Société de l'Information) le dernier projet de loi en date porte sur «Création et Internet » et est plus communément appelé loi Hadopi (Haute Autorité pour la Diffusion des Euvres et la Protection des droits sur Internet) aujourd'hui nommée Hadopi 2. Il prévoit notamment un système de riposte graduée pour l'internaute téléchargeant illégalement ainsi que la mise en place d'une structure de surveillance.

(4) Le crowdsourcing est assimilé à la production collective de biens par des communautés d'internautes. De nombreux exemples de sites font appel à la «foule » pour développer leur contenu comme par exemple Wikipédia dont les articles sont rédigés directement par les internautes. 
Par ailleurs, la propriété intellectuelle constitue de plus en plus le fondement de positions stratégiques offensives plutôt que défensives. En effet, audelà du simple moyen de protection juridique des inventions afin de ne pas se faire copier, la propriété intellectuelle devient le vecteur de stratégies agressives passant par le jeu des licences, par la valorisation des actifs immatériels, par l'utilisation des cours de justice pour empêcher un concurrent d'exercer. Cette vision de la propriété intellectuelle n'est pas uniquement le fait des seules entreprises. Elle est partagée également par des acteurs plus politiques ou institutionnels, qui envisagent la propriété intellectuelle comme une arme de compétitivité économique. Le renforcement de la protection des innovations dans les secteurs du vivant et des TIC (Technologies de l'Information et de la Communication) a, dans ce contexte, été considéré comme une voie privilégiée à emprunter. Ce renforcement généralisé de la propriété intellectuelle pour un bon nombre de secteurs et le relâchement des

critères de brevetabilité sont apparus tant au niveau des États (États-Unis, Europe et Japon) qu'à un niveau plus international au travers de traités tels que les accords ADPIC.

Plusieurs éléments ont contribué à cette tendance et, combinés les uns aux autres, ils ont conduit à placer aujourd'hui la propriété intellectuelle au cœur des questions d'innovation mais aussi des stratégies d'acteurs. Le contexte institutionnel (1.1), les stratégies d'acteurs autour de l'accumulation des brevets (1.2), la transformation du droit pour répondre aux attentes du monde numérique (1.3), ont contribué à faire évoluer dans le temps les régimes de propriété intellectuelle et à les durcir.

\subsection{Rôle crucial du contexte institutionnel et protection de nouveaux domaines techniques}

La tendance au renforcement des droits de propriété intellectuelle est devenue progressivement une question de fond, car elle concerne des objets soit complètement nouveaux soit existants déjà mais protégés jusqu'alors de manière différente. Par exemple, l'usage du brevet comme moyen de protection va peu à peu toucher le monde des logiciels (alors couvert par le droit d'auteur) et va aussi s'étendre au domaine du vivant (brevetage des gènes). L'avènement d'Internet ainsi que la numérisation et la dématérialisation de biens informationnels désormais marchandisables vont de leur côté remettre en question la protection du droit d'auteur ou des marques.

Toute une série de changements institutionnels accompagnent ce bouleversement, d'ordre jurisprudentiel, législatif ou relativement à des traités internationaux. Il est aujourd'hui admis par exemple que les accords ADPIC signés dans le cadre des accords de l'OMC de 1994 ont été le signal emblématique d'un usage renforcé de la propriété intellectuelle tous azimuts (Remiche, 2002). Il est aussi bien montré dans une littérature récente que les États-Unis 
ont eu un rôle pivot dans ce mouvement, initiant les premiers changements dans le domaine du vivant (5) (Bayh Dole Act de 1980) (Orsi, 2002).

Concernant la brevetabilité des logiciels, ce sont les décisions jurisprudentielles qui en ont été à l'origine. Le premier tournant décisif aux États-Unis date de 1980 lors de l'affaire Diamond v./Diehr (6). En Europe, à travers trois décisions clés prises par la chambre des recours de l'OEB (Office Européen des Brevets) (7), un programme peut obtenir une protection par le brevet si il y a effet technique, qu'il soit direct ou potentiel (Schwarz, 1997). Toutefois, même si l'acceptation du brevet logiciel est chose faite aujourd'hui (malgré encore quelques atermoiements en Europe), il reste un domaine dans lequel les points de vue divergent: les méthodes commerciales. Ce terme correspond à un certain nombre d'outils aidant à «faire du commerce ». Aux États-Unis, ce vocable est utilisé pour beaucoup d'applications aujourd'hui brevetables: des méthodes de management, des méthodes de traitements de données financières, ainsi que des techniques mises en œuvre informatiquement, des méthodes éducatives, d'organisation, de commerce électronique, de conseil, de marketing ou financières. S'agissant du commerce électronique, on trouve des brevets sur des systèmes de e-finance, de vente de livres en ligne, d'organisation d'enchères (Lerner, 2000; Hall, 2003). L'analyse juridique montre que les méthodes commerciales ne sont pas traitées de la même manière aux Etats-Unis et en Europe, les premiers étant favorables à une protection par brevet tandis que les seconds le considèrent du ressort des idées (Liotard, 2004). Le cadre européen se démarque du cadre juridique américain puisque l'OEB n'accorde pas de brevet sur les méthodes commerciales et que le critère retenu est celui de l'effet technique (et non celui de l'utilité). Par sa décision Pension Benefits System Partnership en date du 8 septembre 2000 (8), l'OEB a, en effet, sur la base de ce même critère de technicité, refusé de considérer

(5) Bien plus, dans le domaine des biotechnologies, les évolutions dans le champ de la propriété intellectuelle et l'acceptation de brevets sur les gènes ne sont pas les seuls changements d'importance contribuant à l'essor du secteur. Le poids de la finance (capital-risque, Nasdaq ouvert aux start-up) complétera le nouveau régime de PI pour marchandiser la connaissance (Coriat et Weinstein, 2008).

(6) Dans cette affaire il s'agissait de savoir si un brevet pouvait être accordé sur un procédé de séchage du caoutchouc, au moyen d'un logiciel vérifiant la température grâce à une équation mathématique. La Cour suprême des États-Unis, sollicitée par l'USPTO (US Patent \& Trademark Office) estima que dans son ensemble l'invention concernait en ellemême le processus de moulage du caoutchouc et qu'elle était de ce fait brevetable. Elle considéra donc qu'une revendication pouvait être brevetable même si elle utilisait un algorithme mathématique dans le processus puisque l'inventeur ne revendiquait pas tous ses droits pour les futurs usages de l'équation mais seulement une application particulière qu'il avaient inventée.

(7) Décision Computer-related invention/Vicom, T208/84 (1987) JO EPO 14; General purpose management system/Sohei, T769/92 (1995) JO EPO 525; Computer program product/IBM, T 1173/97 (1999) JO EPO 10. 
qu'une pure méthode financière informatisée puisse être considérée comme brevetable (Warusfel, 2003).

\subsection{Accumulation de brevets et stratégies d'acteurs}

Cette situation conduit alors à une explosion du nombre de brevets dans certains secteurs, amenant les détenteurs à concevoir des stratégies autour de leur portefeuille afin de le valoriser au mieux, dans un contexte où la $R \& D$ est de plus en plus coûteuse et complexe (cf. le cas des procès pour gêner un concurrent (9); les stratégies de patent trolls (10), les comportements de vente et de revente de noms de domaine).

D'un point de vue théorique, la multiplication des brevets sur les innovations peut conduire à une situation qualifiée d'anticommons (Heller et Einsenberg, 1998). On assiste à une fragmentation excessive de la propriété des innovations entre un trop grand nombre d'acteurs, impliquant alors une grande difficulté à savoir qui fait quoi, et qui possède quoi. Cette parcellisation extrême de la base de connaissance implique une identification plus difficile des propriétaires, d'autant plus si on souhaite négocier des licences. Face à cette multipropriété, il devient complexe, voire impossible pour les acteurs intéressés à l'implémenter, d'obtenir dans leur globalité des licences. Comme le souligne Foray (2002) «Lorsqu'on octroie des droits privés sur des fragments de gène avant que le produit correspondant ne soit identifié, personne n'est en mesure de regrouper ces droits - obtenir des licences - ce qui empêche le développement du produit ». Dans cette configuration, l'innovation, qui au départ doit être favorisée par le système de droits de propriété intellectuelle, s'en trouve affaiblie. "Si l'on accorde trop de droits de propriété aux micro-composants de l'espace informationnel, l'exploration s'en trouve alors extrêmement coûteuse, voire impossible, parce que l'explorateur doit sans cesse négocier et acheter des droits d'accès. Nous sommes ici confrontés à un vaste paradoxe: généralement utilisés pour soutenir l'exploitation du savoir, les droits de propriété intellectuelle finissent par devenir un instrument de la réduction de la

(9) Les procès récents que Nokia et Qualcomm se sont intentés mutuellement pour infraction autour des brevets de la $3 \mathrm{G}$ attestent de la volonté des protagonistes de peser sur le prix des licences de ces brevets et donc de passer par des menaces et des procès pour gêner l'autre (Liotard, 2008).

(10) La firme agissant ainsi tente de poursuivre une autre firme pour infraction aux revendications de ses brevets et donc l'incite par ce moyen de pression à conclure un accord avec elle (vente et achat de licence). La firme agissant ainsi fait de l'utilisation de la concession de licence et des litiges un réel business model. Deux formes de «trolling » existent: (i) on obtient un brevet pour une innovation sans intention de la produire ou de l'exploiter, en espérant attraper une firme qui utiliserait cette innovation ultérieurement ; (ii) on développe un portefeuille de brevets dans le but soit de vendre plus tard des brevets à un prix élevé ou de les utiliser sur la base de procédures juridiques. Cette stratégie cause des dommages surtout dans les technologies complexes et interopérables en raison de la facilité d'avoir un brevet bloquant le système (OEB, 2007). 
base de connaissances » (page 85). La biotechnologie est l'apanage d'une telle fragmentation. Le passage par une mutualisation de la PI sous la forme d'un patent pool (communauté de brevets) commence à faire son chemin auprès des acteurs de la biotechnologie, qui y voient une solution pour diminuer les coûts de transaction, éviter les brevets de blocage ou les licences en cascade (Clarke et alii, 2001).

Le secteur des TIC (Technologies de l'Information et de la Communication) utilise ainsi de telles organisations assez fréquemment. La raison en est simple. La complexité grandissante des technologies, la convergence de secteurs qui historiquement étaient cloisonnés, le caractère cumulatif des innovations (Merges et Nelson, 1994) et l'impulsion des technologies numériques ont conduit à mettre sur le marché des produits dont la propriété est de plus en parcellisée. Aujourd'hui pour une même technologique, force est de constater la présence de multiples firmes qui, chacune, possède un bout de la technique. Les règlements des questions portant sur la PI sont d'autant plus importants dans ces domaines que la bonne gestion de celle-ci conditionnera aussi la mise en place de standards technologiques. L'exemple du MPEG2 (Moving Picture Expert Group) illustre parfaitement cette situation. Cette technologie de compression vidéo couvre aujourd'hui plus de 600 brevets répartis entre une vingtaine de propriétaires. La solution consistant à regrouper la gestion de ces droits au sein d'une structure dédiée (nommée MPEG LA) qui propose une licence unifiée de la technologie auprès des licenciés, permet ainsi de répondre au problème de l'accumulation des brevets et des problèmes d'accès des tiers. MPEG 2 n'est pas le seul exemple de création de patent pool.

\subsection{Le droit à l'épreuve de la régulation en matière de biens informationnels}

La dématérialisation des œuvres via des supports électroniques entraîne de nouvelles interrogations sur la façon de réguler juridiquement ces biens informationnels : faut-il appliquer les règles traditionnelles de la propriété intellectuelle ou bien faut-il penser à des formes nouvelles et particulières de protection pour la diffusion de ces œuvres? Les droits d'auteur d'une part et la propriété industrielle (brevet, marques ou dessins et modèles) d'autre part s'inscrivent dans ce débat dont l'issue a conduit à certains résultats comme par exemple le traité de l'OMPI (Organisation Mondiale De la Propriété Intellectuelle) du 20 décembre 1996 reconnaissant la portée du droit d'auteur sur les réseaux numériques, ou bien l'apparition de sanctions judiciaires à l'encontre des actes nombreux de cybersquatting sur les marques (voir la deuxième partie de cet article). Dans d'autres domaines technologiques en revanche des solutions nouvelles ont été apportées. C'est le cas de la protection des bases de données en Europe qui propose un droit sui generis (11) depuis la

(11) Un droit sui generis est un droit qui s'applique à un objet particulier, ici les bases de données. 
directive communautaire du 11 mars 1996, au sein duquel on a construit hors du champ même de la propriété intellectuelle un droit des producteurs destiné à préserver uniquement la pérennité économique de ses investissements contre les différentes formes de parasitisme (Warusfel, 2004).

Par ailleurs, à l'heure d'une meilleure compréhension par les firmes de la valorisation de leur patrimoine immatériel et technologique, l'apparition de nouveaux canaux de diffusion et la numérisation croissante des biens informationnels rendent compliquée la préservation des droits et, plus encore, nécessitent une réflexion sur la cohérence des critères classiques de protection avec les besoins nouveaux des entreprises. Plus précisément, un décalage entre la réalité technique contemporaine et l'état des textes de droit suscite de nombreux débats.

C'est particulièrement le cas s'agissant de la protection des inventions logicielles. L'invention logicielle est traditionnellement protégée par le droit d'auteur (ou le copyright dans les pays anglo-saxons) moyennant quelques particularités liées à la qualité de ce bien en matière de reproduction et de copie et aux questions de décompilation (Liotard, 2002). Mais depuis plus d'une vingtaine d'années on observe en Europe et aux États-Unis un mouvement de fond ayant pour objectif de protéger le logiciel par le brevet et donc d'accorder aux inventeurs une protection différente de celle proposée par le droit d'auteur. Le brevet logiciel protège les interrelations fonctionnelles entre les composants techniques du système. Dès lors, ce qui est interdit est la reproduction de ces revendications : il n'est plus question de protéger la forme du logiciel mais plutôt de protéger une série de fonctions c'est-à-dire un résultat. L'observation de la jurisprudence américaine en la matière montre par exemple que les inventions logicielles, si elles répondent aux critères classiques de brevetabilité de l'USPTO (non-évidence, nouveauté et utilité) sont susceptibles d'être protégées par brevet comme toute autre invention conformément aux dispositions du Patent Act. Les dernières barrières en matière de brevetabilité logiciel sont tombées en 1998 avec la décision State Street Bank contre Trust Co v./Signature Financial Group Inc. Cette décision a ouvert la voie à la brevetabilité des méthodes commerciales ou business methods (Liotard, 2004). Cette tendance à une extension tous azimuts de la protection par le brevet n'a pas totalement été reprise par l'Europe, qui demeure pour le moment plus contraignante que les Etats-Unis, en ce qui concerne particulièrement le traitement des méthodes commerciales. S'agissant du logiciel, l'exclusion littérale des programmes d'ordinateur en tant que tels de la brevetabilité est inscrite dans les lois de brevet (notamment en France, dans l'article L. 611-10 CPI) et dans la Convention de Munich (article 52), donnant l'impression aux innovateurs qu'en matière de logiciel seules les règles du droit d'auteur peuvent s'appliquer à leurs innovations, alors que dans les faits, de nombreux brevets sur les logiciels sont déposés en Europe, comme en témoigne la pratique de l'Office européen des Brevets depuis vingt ans. 
On constate alors qu'après une période de diffusion des innovations via un système de PI assez souple (Jaffé, 2000), les années 80 marquent un tournant vers un renforcement du système de brevet, une protection plus sévère des innovations logicielles, une diffusion plus difficile et des acteurs utilisant la PI comme un outil stratégique. C'est ce contexte durci de la protection qui va provoquer la contre-attaque du logiciel libre. C'est aussi ce contexte initié par les États-Unis qui va leur permettre de remettre la main sur certains secteurs, et de doper leur compétitivité. On assiste ainsi à un changement de régime de PI. Ce terme introduit par Teece (1986) fait directement référence à la littérature sur les systèmes forts ou faibles de PI et sur les effets de tel ou tel renforcement du brevet sur l'innovation (12). L'existence d'un régime de propriété n'est pas sans conséquence sur les choix d'acteurs dans leur manière de « jouer » sur le marché avec la PI (13).

Ce changement de régime a conduit à une littérature abondante sur ce thème (Jaffé, Dosi et alii, 2006) portant sur une question cruciale: est-ce qu'un système fort de PI est nécessaire pour l'ensemble des secteurs? Constitue-t-il ou non un catalyseur du rythme de l'innovation? Un système faible de PI dans lequel l'imitation est permise n'est-il pas la meilleure solution? Dosi et alii (2006) recensent ces questions et concluent qu'il n'y a pas de lien marqué et évident entre davantage de PI et davantage d'innovation (14).

Le développement d'Internet et l'expansion de nouveaux services et sites sur la toile ont une influence directe sur les règles traditionnelles de la PI et en redessinent les contours selon le type d'objet proposé et diffusé. Les règles qui entouraient par exemple le droit d'auteur sont aujourd'hui bouleversées par la montée en puissance de biens informationnels, dématérialisés de leur contenant et vendus en tant que tels (musique, film, livre, etc.). À l'heure de l'avènement de l'économie numérique et d'Internet, l'observation des stratégies de firmes permet un constat simple: le passage vers une Nouvelle Économie n'a fait que renforcer et accentuer l'utilisation, voire l'usage stratégique, de la propriété intellectuelle par les acteurs économiques.

Cet environnement global vers davantage de PI a un effet important sur la manière dont l'adressage sur Internet est appréhendé aujourd'hui par les acteurs économiques. Nous abordons ce cas d'application dans la partie suivante dans laquelle nous porterons notre attention sur le cas d'un nouveau

(12) Voir le débat théorique opposant Kitch (1977) et Scotchmer (1991), Merges et Nelson (1994) sur l'octroi d'une protection forte ou non au premier innovateur.

(13) L'exemple type d'un secteur dans lequel l'appropriation est forte est celui de la pharmacie où le recours au brevet s'avère vital, pour protéger une innovation largement copiable et dans laquelle le reverse engineering est possible (Levin et alii, 1987).

(14) Le logiciel tout particulièrement a fait l'objet d'une analyse approfondie (Bessen et Maskin, 2000). 
marché autour des noms de domaine, le système de revente ainsi que les mécanismes de résolution des conflits autour de la PI et particulièrement des marques.

\section{II. - LA PROPRIÉTÉ INTELLECTUELLE AU CENTRE DES MODALITÉS DE MARCHANDISATION DES NOMS DE DOMAINE SUR L'INTERNET}

Les noms de domaine étaient à l'origine uniquement destinés à faciliter la mémorisation des adresses et la navigation sur le réseau internet. Ils sont devenus l'objet de stratégies de valorisation marchande relativement complexes (existence d'un premier et d'un second marché, d'une offre concurrentielle de l'enregistrement et d'une demande parfois purement spéculative, de multiples litiges qu'il faut arbitrer etc.). Nous montrerons dans un premier temps comment s'est opérée la «marchandisation » de lignes de codes informatiques qui sont devenues un quasi équivalent de marques commerciales. Dans un second temps, nous verrons que cette marchandisation a conduit à l'émergence d'une industrie opérant sur plusieurs marchés de l'enregistrement et de la gestion des noms de domaine, marchés dont nous analyserons les principales modalités de régulation. Enfin, et dans un troisième temps, nous examinerons plus particulièrement les modalités de règlements des conflits, liés à la propriété intellectuelle, qui sont de plus en plus fréquents dans cette activité.

\subsection{Le processus de marchandisation de l'adressage d'Internet}

Les noms de domaine ont initialement été créés pour permettre une mémorisation rapide de la localisation (adresse) des premiers ordinateurs mis en réseau. Chaque ordinateur est identifié et localisé par une adresse (adresse IP pour Internet Protocol) qui se présente sous la forme d'une suite de quatre séries de nombres allant de 0 à 255 (15). Comme pour les numéros de téléphone, chaque adresse IP doit être unique et chaque nom de domaine, qui en est son double sous forme de nom, doit donc également l'être. Cette contrainte d'unicité nécessite donc la tenue d'un répertoire (dit registre) des noms de domaine. Avec l'augmentation du nombre d'ordinateurs mis en réseau, un système automatique de traduction des adresses IP en noms de domaine a progressivement été mis en place et est devenu en 1983 le DNS (Domain Names System) (Le Mire 2003).

La marchandisation du système des noms de domaine résulte de deux évolutions parallèles: la première est le développement très rapide d'Internet et la

(15) Les besoins croissants d'adresses conduisent à la mise en place de nouveaux protocoles techniques reposant sur des adresses de plus en plus longues qui permettraient un nombre quasi infini d'adresses (IPV6). 
nécessité d'une gestion de plus en plus professionnelle du DNS et la seconde est la croissance des relations commerciales sur le réseau qui a conduit à la valorisation des noms de domaine de second niveau.

L'augmentation exponentielle des ordinateurs reliés au réseau internet a conduit à la mise en place d'une véritable industrie de l'enregistrement des adresses sur le DNS. La structure de cette industrie a évolué, passant d'un monopole à une ouverture notable à la concurrence avec la séquence suivante. En 1992, le gouvernement américain passe un contrat avec Network Solution Inc. (NSI) qui est une organisation à but non lucratif de droit privé, pour assurer le contrôle et la gestion des noms de domaine sous le contrôle de la NSF (National Science Foundation). Pour financer ces activités, NSI est autorisée à percevoir une rémunération pour l'inscription de chaque nom de domaine. Cette rémunération est de 100 dollars par an pour les deux premières années d'enregistrement puis 50 dollars par année suivante. Un embryon de marché de l'enregistrement des noms de domaine naît donc sous la forme d'un monopole très rentable. En effet, le coût de l'enregistrement d'un nom de domaine est très faible puisqu'il s'agit d'ajouter quelques lignes de codes informatiques dans le DNS. La situation monopolistique de NSI est de plus en plus contestée par l'Administration des États-Unis et en particulier par le Département du Commerce qui impose à partir de 1997 la mise en concurrence de l'enregistrement des noms de domaine. En 1998, l'administration américaine confie à un organisme à but non lucratif de droit privé, l'ICANN (l'Internet Corporation for Assigned Names and Numbers) la gestion du système des noms de domaine. Le monopole de NSI prend fin avec la possibilité pour des sociétés accréditées par l'ICANN de gérer l'enregistrement des noms de domaine. À partir de cette date, l'ICANN contrôle le processus de création des noms de domaine de premier niveau (à l'extrême droite de l'adresse comme.com) d'Internet mais ne gère pas directement le registre. Cette fonction est dévolue à d'autres firmes qui ont obtenu de l'ICANN le monopole de la gestion des noms de domaine de premier niveau (comme Verisign pour le «com» et le «net») et exercent la fonction dite de « registre » (ou registry). Les registres délèguent la création et la gestion des noms de domaine de second niveau (à gauche du nom de premier niveau) à des « bureaux d'enregistrement » (ou registrar). La concurrence dans l'enregistrement des noms de domaine n'apparaît donc qu'à partir des noms de second niveau (Simon 2007).

Le processus de marchandisation des noms de domaine est historiquement lié au développement très important des noms de domaine de second niveau et aux enjeux commerciaux qui découlent de leur possession. Les noms de domaine de second niveau attirent du trafic sur l'Internet (renault « com » ou sex «com ») et peuvent posséder une valeur commerciale très largement supérieure à leur coût d'acquisition (en fait de location de l'ordre de quelques dizaines d'euros par an) auprès des bureaux d'enregistrement. En outre, l'enregistrement d'un nouveau nom de domaine s'effectue selon la règle du «premier demandeur - premier (et seul) servi ». Les débuts de la marchandisation des noms de domaine de second niveau ont en particulier permis la réalisation 
de profits importants pour des personnes averties qui avaient acheté des noms « désirables » pour les revendre beaucoup plus chers, ceci qu'il s'agisse de noms de marques, de personnes ou de noms génériques «valorisables ».

Les noms de domaine de second niveau sont donc de plus en plus considérés comme quasi équivalents à des marques et possèdent une valeur marchande qui peut, comme nous le verrons, être importante. Leur multiplication a engendré la création et le développement d'une véritable industrie de l'enregistrement dont nous présentons la structure et les comportements ci-dessous.

\subsection{Structure et comportement de l'industrie de l'enregistrement des noms de domaine}

Il existe actuellement environ 270 noms de domaine de premiers niveaux (ou TLD pour Top Level Domains ou extensions). Parmi ceux-ci, environ 250 noms de domaine correspondent à des pays ou des territoires géographiques (ccTLD) et 21 sont dits " génériques », (gTLD) comme par exemple les.com.org ou.aero. Le marché de l'enregistrement qui s'inscrit au sein de ce niveau opère donc sur une base très importante et internationale.

Il faut en fait distinguer deux marchés de l'enregistrement qui fonctionnent de manière très différente :

— le marché du premier enregistrement est ponctuel et particulièrement actif lorsqu'un nouveau nom de premier niveau (ou extension) est créé. Le premier enregistrement des noms de second niveau se fait moyennant un prix relativement faible en raison de la concurrence existant entre les bureaux d'enregistrement ;

- le marché de la revente (second marché) fonctionne en continu et les prix peuvent, pour certains noms, être très élevés. Il concerne essentiellement la revente de noms de second niveau (16). Nous centrerons l'analyse de ce marché sur l'extension en «.com » qui est de loin la plus importante (figure 1). Rappelons que la gestion de l'extension (nom de domaine de premier niveau) «.com »a été confiée par l'ICANN à la firme américaine Verisign qui fait fonction de registre et que les adresses sont enregistrées par de multiples bureaux d'enregistrement.

\section{Le marché de premier enregistrement}

En 2009, il existait dans le monde un peu plus de 80 millions d'adresses en «.com. » et leur enregistrement est réalisé par plus de 900 bureaux d'enregis-

(16) On assiste à l'émergence d'un marché pour le troisième niveau (à nouveau vers la gauche de l'adresse comme par exemple drink.coca-cola.com) mais celui-ci reste encore embryonnaire (C. Manara 2007). 
FIGURE 1 : Classement des noms de domaines

( $G$ pour générique et $P$ pour pays) en millions

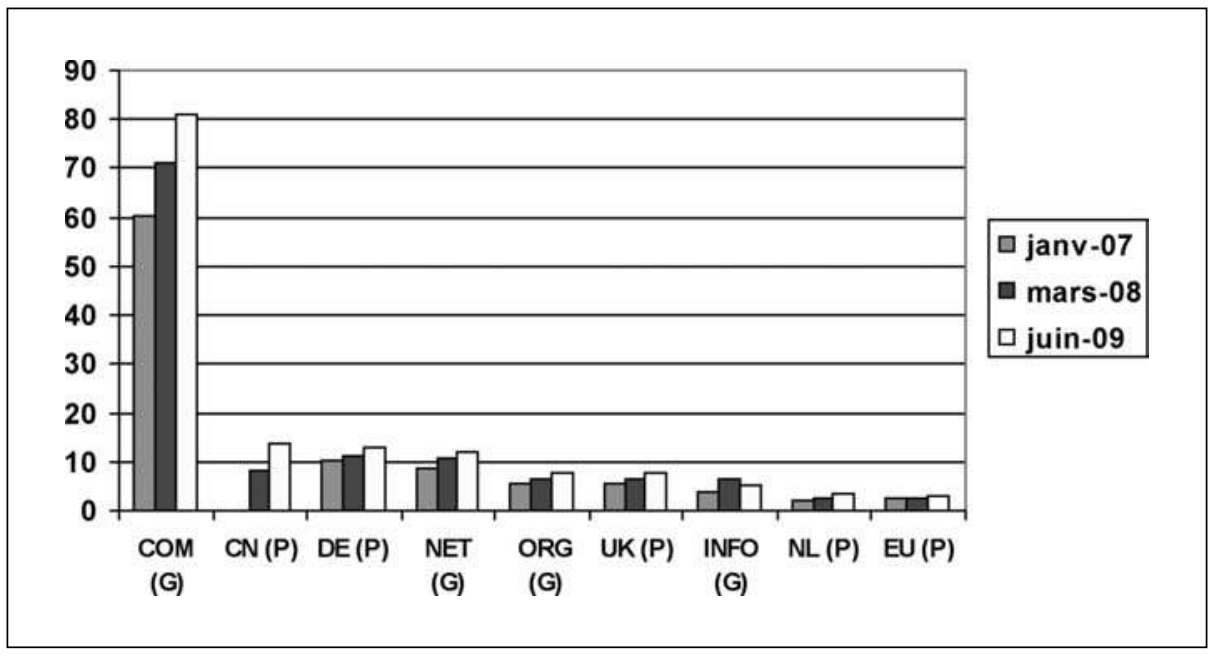

Source : d'après DomainIfo 2008

trement. La structure de cette industrie des bureaux d'enregistrement se présente sous la forme d'un oligopole avec une frange de multiples bureaux d'enregistrement de taille modeste. Les principaux bureaux d'enregistrement (plus d'un million d'adresses enregistrées par an) représentent $64,8 \%$ de l'activité, les quatre premières $47,1 \%$ et la firme leader: Go Daddy $22 \%$ (DomainInfo2008).

L'ouverture à la concurrence au niveau des bureaux d'enregistrement a conduit à l'émergence de nouveaux acteurs et à une forte réduction des prix. Ainsi, le prix annuel pour l'enregistrement d'un nom de domaine en «com » qui était de 50 \$ en 1998 est en dessous de 15 \$ en moyenne en 2008. Par ailleurs, la part de marché de l'enregistrement pour l'ex-monopole NSI (devenu NS LLC) est passée à moins de $6 \%$ en 2007.

Les modalités d'enregistrement des noms de domaine de second niveau ont connu des évolutions importantes pour tenir compte du respect des marques et des propriétaires avérés de noms ayant une certaine notoriété. Lors du lancement d'un nouveau nom de domaine de premier niveau (comme par exemple le « eu » en 2006) une période de prélancement (sunrise) permet aux titulaires de marques reconnues d'enregistrer leur nom dans ce nouveau domaine afin d'éviter l'achat du nom par un tiers qui le leur proposera à la revente moyennant un profit important (cybersquatting). Toutefois, et comme nous le verrons plus loin, ce mécanisme de protection n'empêche pas l'existence de comportements prédateurs lors de toute campagne d'introduction de nouveaux noms de domaine. 
Une réforme importante est en cours concernant la création de noms de domaine de premier niveau. L'ICANN a annoncé en 2008 que le lancement de nouveaux noms de domaine serait totalement libéralisé à partir de 2011. Jusqu'à présent, la création de nouveaux noms de domaine génériques de premiers niveaux était très peu courante car il était très difficile et très long de convaincre l'ICANN de l'utilité d'un nouveau nom. À partir de 2011, l'ICANN autorisera le lancement de toute nouvelle extension à condition que le projet soit techniquement et financièrement viable. On devrait donc assister à une prolifération de nouvelles extensions créées par exemple par des villes (.paris) ou des entreprises (.danone). Il est encore très difficile de savoir, au milieu de 2009, combien de nouveaux noms vont apparaître mais il est probable qu'ils se compteront par centaines ou milliers. Les grandes entreprises devraient par exemple être fortement incitées à créer leur extension afin de la protéger, ceci malgré la barrière à l'entrée d'un prix de dépôt d'un dossier auprès de l'ICANN de $185000 \$$. Ce processus est très critiqué, notamment par les entreprises qui craignent, à juste titre, une augmentation des coûts de protection de leur marque proportionnelle au nombre d'extensions créées et aussi l'augmentation des conflits. Une expertise menée à la demande de l'ICANN par un spécialiste de l'économie de la concurrence (Carlton 2009 a et b) et favorable à la libéralisation fait l'objet d'un certain nombre de critiques (Kende 2009, SIAA 2009).

\section{Le marché de la revente ou second marché}

Il existe, parallèlement au marché ponctuel de l'enregistrement des nouveaux noms de domaine un marché de la revente (second marché ou marché de l'occasion) qui fonctionne de manière continue et passe par des ventes de gré à gré publiques ou non ou par des enchères. L'achat de noms de domaine pour des raisons spéculatives alimente aussi ce second marché. La plupart des noms de domaine qui font l'objet de transactions ont une certaine popularité qui leur donne une valeur marchande. Dans certains cas, ils ont été achetés pour gêner une entreprise ou un individu et l'obliger à en négocier le rachat. Le second marché des noms de domaine est donc un marché de l'occasion mais, contrairement aux autres biens, les prix de revente sont plus élevés que les prix d'achat initiaux. Cette particularité s'explique par l'existence de différences fondamentales dans les modalités de fixation des prix. Lors du premier enregistrement, le prix est faible en raison de la concurrence existant entre les centaines du bureau d'enregistrement qui vendent aux internautes des noms qu'ils enregistrent moyennant un prix fixe reversé à des registres. Les prix de revente dépendent uniquement de l'attractivité du nom dont le propriétaire possède un monopole, il peut donc être très élevé pour des noms qui peuvent attirer beaucoup de trafic de la part des internautes. Le rachat de noms de domaine peut se faire de manière directe si un internaute constate qu'il n'a pas été renouvelé et est tombé dans le domaine public. (Il s'agit parfois d'un simple oubli). Toutefois, le rachat devient de plus en plus une activité « industrielle » et des entreprises se sont spécialisées dans le suivi de la retombée dans 
le public des noms de domaine et se constituent un stock de noms qu'elles exploitent ou revendent (17).

Bien que des données précises concernant le niveau des transactions sur le second marché ne soient pas disponibles de manière publique, un certain nombre d'informations donne une idée de l'importance de certaines d'entre elles. La revente publique la plus élevée de l'histoire a eu lieu en 2006 avec « sex.com» pour 14 millions de dollars. Le nom «business.com» a été vendu 7,5 millions de $\$$, « worldwideweb.com » a été vendu pour 3,5 millions de $\$$ et « vodka.com » pour 3 millions de $\$$. Ces montants sont spectaculaires mais très rares, la valeur médiane (calculée d'après les données de DNJournal.com) du prix de revente des 100 plus importantes ventes en 2008 était de 176000 \$, en baisse par rapport à 2009. Les ventes de noms sous l'extension générique.com sont de loin les plus importantes et enregistrent les prix les plus élevés.

\subsection{Propriété intellectuelle et régulation des marchés des noms de domaine}

La valeur d'un certain nombre de noms de domaine de second niveau peut être due à la présence d'une marque qui attire les internautes directement ou via les moteurs de recherches. Pour d'autres noms, c'est la présence d'un terme générique très populaire qui en explique la valeur. Toutefois, la constitution de la valeur d'un nom de domaine est différente de celle d'une marque (Wang 2006). La valeur d'un nom de domaine est liée au développement d'Internet et notamment de son internationalisation. Elle est d'autant plus forte que le nom est évocateur pour un nombre le plus important possible d'internautes (exemple du nom « sex.com »). En revanche, la valeur commerciale d'une marque provient en grande partie d'une spécificité et d'une notoriété qui s'est construite dans le temps. Un nom de domaine peut bénéficier de cette notoriété mais ne peut pas la créer à court terme. Toutefois, les problématiques de protection et de régulations des conflits sont proches et ont conduit à la mise en place d'instances et de mécanismes de régulation coordonnés entre le droit de la propriété intellectuelle et le droit d'Internet.

Des conflits liés à des questions de propriété intellectuelle ont émergé dès le début de l'histoire d'Internet commercial. Le coût particulièrement faible de l'enregistrement des noms de domaine a permis à des initiés d'enregistrer des noms célèbres et il était difficile au propriétaire de ce nom de le récupérer (le cybersquatting). D'autre part, certains noms identiques pouvaient exister pour des produits différents et il était difficile d'arbitrer entre des entreprises de bonne foi (cas des produits laitiers et des stylos Montblanc). Enfin,

(17) En 2006, l'OCDE a consacré une étude au marché secondaire des noms de domaines, en a estimé la taille et montré la dynamique au sein d'une offre relativement concentrée (OCDE 2006). 
s'agissant d'un réseau mondial, les procédures de protection et de récupération pouvaient être très longues et très coûteuses. Le phénomène du cybersquatting s'est développé dès le milieu des années quatre-vingt-dix et les titulaires de marques ont rapidement demandé et obtenu qu'un mécanisme approprié de résolution des conflits soit mis en place dans le cadre de la création de l'ICANN (Defossez, 2006). Celle-ci, en liaison avec l'OMPI (Organisation Mondiale de la Propriété Intellectuelle) a mis en place en 1999 un mécanisme spécifique de résolution des conflits pour les noms de domaine: l'UDRP (Uniform Domain Name Dispute Resolution Policy). Ce mécanisme est plus rapide et moins onéreux que la voie légale traditionnelle car il repose sur un principe proche de l'arbitrage. Il n'est d'ailleurs pas étonnant de constater que, comme la régulation du réseau physique d'Internet qui passe par des accords privés, la résolution des conflits sur les noms de domaine se fasse en dehors du système juridique traditionnel. Les titulaires des noms de domaine doivent respecter les décisions de l'arbitre en cas de conflit et c'est le bureau d'enregistrement qui opère la modification dans sa base de données en supprimant le squatter.

Les procédures de l'URDP s'appliquent pour tous les noms de domaine génériques. Pour les noms de domaine des pays ou des zones géographiques, il existe un système très proche mais plus décentralisé. Ce mécanisme spécifique a réduit le cybersquatting classique en simplifiant la résolution des conflits. Le nombre des procédures augmente régulièrement mais, au regard du rythme de croissance des noms de domaine créés, on peut considérer que le nombre de conflits est en baisse relative.

\section{FIGURE 2 : Évolution du nombre de conflits arbitrés par l'URDP}

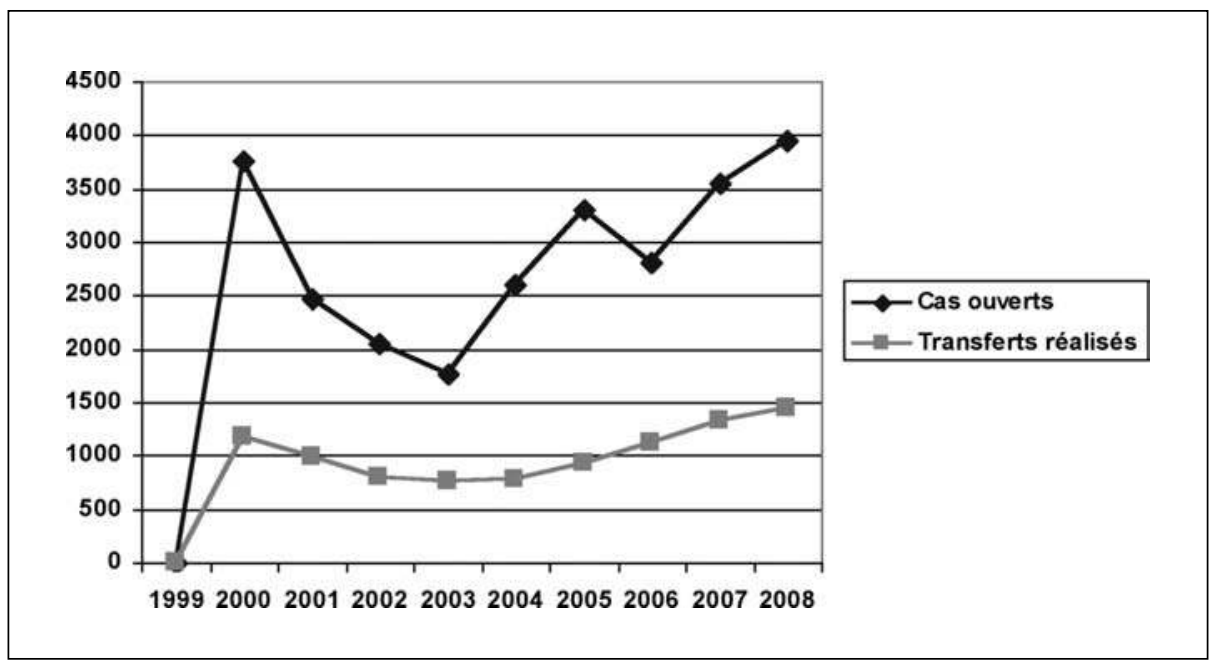

Source: (http://www.wipo.int/amc/en/domains/statistics) 
Une part notable des procédures engagées aboutit à un transfert du nom vers le plaignant ; c'est par exemple le cas pour 1437 dépôts de plainte devant l'URDP de l'OMPI en 2008.

Le cybersquatting classique est donc relativement maitrisé mais il connaît des évolutions allant vers un certain élargissement des pratiques constatées. Il ne concerne plus seulement des marques commerciales mais aussi par exemple des noms de personnalités (personnalités politiques lors d'élections par exemple): on parle de celebrity squatting. D'autres stratégies d'enregistrement exploitent de manière systématique toutes les failles possibles du système des noms de domaine (C. Simon 2007). On assiste à la multiplication de l'inscription de noms de domaine comportant l'ajout d'éléments génériques au nom principal (planetpepsi.com) ou une modification d'une lettre proche de la lettre initiale sur le clavier: c'est ce qu'on appelle le typosquatting. Toutes ces stratégies visent à drainer des cybernautes vers des sites où leur sont proposés des produits ou des services qui contribuent à la valeur marchande de ces adresses. Dans ce cas, la valorisation ne passe pas par la revente du nom acheté mais par la monétarisation du trafic généré. Les internautes qui se retrouvent par erreur sur le site y trouvent des propositions de redirection vers des sites commerciaux et le propriétaire du site initial reçoit une rémunération « au click». Une autre stratégie consiste à récupérer des noms qui n'ont pas été renouvelés, souvent de manière involontaire ou par omission, puis de les revendre au précédent titulaire négligeant.

\section{CONCLUSION}

L'objectif de cet article a été de montrer comment, dans une période récente, les droits de propriété intellectuelle protègent des nouveaux objets numériques liés à Internet et comment finalement les brevets, marques et droits d'auteur sont devenus des actifs incontournables pour les entreprises qui en font aujourd'hui des outils stratégiques, dans un environnement de renforcement généralisé des droits. Ces objets informationnels diffusés et créés sur la toile ouvrent alors de multiples interrogations et problèmes relativement à leur protection, à l'heure où l'internaute a plutôt tendance à les contourner. Le droit a donc été contraint de s'adapter à ce nouveau contexte.

S'agissant du nommage que nous avons étudié, il semble aujourd'hui que le respect des règles de la propriété intellectuelle soit désormais relativement assuré par le mécanisme de l'UDRP. Toutefois, deux phénomènes majeurs devraient modifier le fonctionnement d'Internet et avoir des conséquences sur cet aspect particulier de l'économie numérique. Le premier est la probable multiplication des adresses que nécessitera dans un futur proche le développement d' « Internet des objets » (c'est-à-dire la multiplication des objets reliés à Internet comme par exemple des étiquettes ou des appareils électroménagers). Cette évolution ouvre sans doute de nouvelles perspectives à la marchandisation du nommage. Le second est la libéralisation, à partir de 2010, de la création de noms de domaine de premier niveau qui devrait conduire à modifier les 
possibilités de valoriser de nouveaux noms de domaine de second niveau. En outre, mais il est très difficile d'en appréhender les conséquences, va être autorisée l'ouverture d'extensions dans des alphabets différents (noms de Domaine Internationalisés). On devrait également assister à une multiplication des ouvertures, donc des périodes de pré-enregistrement et il n'est pas évident que les titulaires de marques ou de noms puissent ou aient la volonté de réserver des noms de domaine dans toutes les extensions. Le risque de cybersquatting et donc de conflits risque d'être particulièrement important et on peut donc s'attendre à un développement du recours à l'UDRP dans les prochaines années.

\section{RÉFÉRENCES}

AFNIC (2008), Observatoire du marché des noms de domaine en France, édition 2008.

ARROW K. (1962), Economic Welfare and the Allocation of Resources for Invention, in The Rate and Direction of Inventive Activity, Nelson R. (ed.), Princeton University Press, Princeton.

BARBET P. (2006), « La régulation du réseau Internet », in Barbet P. et I. Liotard (éd.) (2006), Société de l'information: approche économique et juridique, l'Harmattan.

BESEN J. \& MASKIN E. (2000), « Sequential innovation, patents and imitation », Working Paper, $\mathrm{n}^{\circ}$ 00-01, january, Department of Economics, Massachusetts Institute of Technology.

CARLTON D. (2009 a), Preliminary report of Dennis Carlton regarding impact of new gtlds on consumer welfare, Compass lexicon 2009.

CARLTON D. (2009b), Preliminary Analysis of Dennis Carlton Regarding Price Caps for New gTLD, InternetRegistries Compass Lexicon 2009.

CHESBROUGH H. (2006), «Open innovation: a new paradigm for understanding industrial innovation », in Chesbrough H., Vanhaverbeke W. et West J., Open innovation: researching a new paradigm, Oxford University Press.

CLARKE J. et alii (2001), Patent pools: a solution to the problem of access in biotechnology patents?, USPTO.

CORIAT B. et WEINSTEIN O. (2008), IPR regimes, firms and the commodification of knowledge, paper presented at the International Conference « Governing the business enterprise: ownership, institutions and society », Paris, 22-23 mai.

CORTÉS DIÉGUEZ J. (2008), «An analysis of the URDP experience - Is it time for reform? », Computer law \& Security Report, 24 (2008), pp. 349-359.

DAMILAVILLE L. et MAIGRON P. (2008), Gouvernance Internet: fonctionnement et dysfonctionnements, communication au Colloque du CEJEM université Panthéon Assas Paris II Internet et culture: quels enjeux juridiques ?, mercredi 14 mai 2008.

DEFOSSEZ A. (2006), «Conflits entre noms de domaine et marques (renommées): l'approche OMPI », Revue Internationale de Droit Économique, pp. 167-209.

DOMAININFO (2008), (www.domainesinfo.fr/statistics.php).

DOSI G., L. MARENGO, C. PASQUALI (2006), « How much should society fuel the greed of innovators? On the relations between appropriability, opportunities and rates of innovations », Research Policy, 35, 2006, pp. 1110-1121.

FORAY D. (2002b), Propriété intellectuelle et innovation dans l'économie du savoir, ISUMA, printemps.

HALL B. (2003), « Business Methods Patents, Innovation and Policy », Working Paper, 9717, NBER, mai. 
HELLER M. \& EISENBERG R. (1998), « Can patents deter innovation? The Anticommons in biomedical research », Science, vol. 280 .

JAFFÉ A. (2000), « The US patent system in transition: policy innovation and the innovation process », Research Policy, 29, pp. 531-557.

KENDE M. (2009), Assessment of ICANN Preliminary Reports on Competition and Pricing, prepared on behalf of AT\&T.

KITCH E.-W. (1977), « The Nature and Functions of the Patent System », Journal of Law and Economics 20, pp. 265-290.

KLINE D. et RIVETTE K. (2000), Rembrandts in the attic: unlocking the hidden value of patents, Harvard Business School Press.

LERNER J. (2000), Where does State Street lead? A First Look at Finance Patents, 1971-2000, WP 7918, NBER: (http://www.nber.org/papers/w7918).

LE MIRE O. (2003), Le nommage est-il une activité de service public?, mémoire du DESS Droit d'Internet administrations - entreprises, université de Paris 1.

LEVIN R., A. KLEVORICK, R. NELSON, S. WINTER (1987), Appropriating the returns from industrial research and development, Brookings Paperson Economic Activity, Microeconomics, $\mathrm{n}^{\circ} 3$.

LIOTARD I. (2002), « La brevetabilité des logiciels: quelques éclaircissements sur l'évolution jurisprudentielle aux États-Unis », in Revue d'Économie Industrielle, numéro spécial, « Les droits de propriété intellectuelle: nouvelles frontières et nouveaux enjeux », $\mathrm{n}^{\circ} 99,2^{\mathrm{ème}}$ trimestre.

LIOTARD I. (2004), « Les brevets sur les méthodes commerciales: état des lieux et perspectives économiques », Propriétés Intellectuelles, avril 2004, $\mathrm{n}^{\circ} 11$.

LIOTARD I. (2008), « Persistance et intensité des conflits entre normalisation et propriété intel-

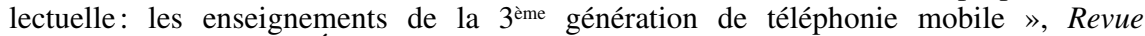
Internationale de Droit Économique, $\mathrm{n}^{\circ} 1$.

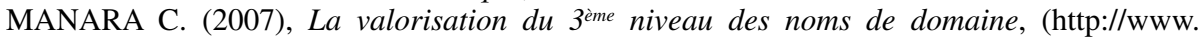
domainesinfo.fr/actualite/1480/chronique-cedric-manara-la-valorisation-du-3eme-niveaudes-noms-de-domaine.php).

MERGES R. et NELSON R. (1994), « On limiting or encouraging rivalry in technical progress : the effects of patent scope decisions », Journal of Economic Behavior and Organization, vol. 25.

MUELLER M., McKNIGHT L. (2004), The post-.COM internet: toward regular and objective procedures for internet governance, Telecommunications Policy 28 (2004) 487-502.

MUELLER M. (2002), Ruling the Root: Internet Governance and the Taming of Cyberspace. Cambridge, MA: MIT Press.

OCDE (2006), Le marché secondaire des noms de domaine, DSTI/ICCP/TISP (2005) 9/FINAL.

OEB (2007), Scenarios for the future; how might IP regimes evolve by 2025 ?

OMPI (2008), Règlement de litiges pour le XXI ème siècle Centre d'arbitrage et de médiation de l'OMPI, (http://arbiter.wipo.int).

ORSI F. (2002), « La constitution d'un nouveau droit de propriété intellectuelle sur le vivant aux États-Unis: origine et signification économique d'un dépassement de frontière », Revue d'Économie Industrielle, $\mathrm{n}^{\circ} 99,2^{\text {ème }}$ trimestre, pp. 65-86.

REMICHE B. (2002), « Révolution technologique, mondialisation et droit des brevets », Revue Internationale de Droit Économique, 2002, $\mathrm{n}^{\circ} 1$.

SCHWARZ T. (1997), «Évolution sur la question de la brevetabilité des logiciels en droit européen des brevets », Droit de l'informatique et des télécoms, $\mathrm{n}^{\circ} 2$.

SCOTCHMER S. (1991), «Standing on the shoulders of giants: cumulative research and the patent law », Journal of Economic Perspective, vol. 5, $\mathrm{n}^{\circ}$ 1, hiver.

SEDO (2007), Secondary domain names study.

SIAA (2009), Software and Industry Information Association, Comment on the « Preliminary Report of Dennis Carlton Regarding Impact of New gTLDs on Consumer Welfare », (www.siaa.net).

SIMON C. (2007), Monétisation de l'immatériel: contribution sur les noms de domaine, miméo Atelier internet, École Normale Supérieure de la rue d'Ulm.

TEECE D. (1986), « Profiting from technological innovation », Research Policy, 15(6), pp. 285305. 
TINÉ C. (2008), juin 2008, Quand le nommage internet a changé d'ère, (http://www. mailclub.info/article.php3?id_article=868).

UIT (2005), The Internet of things, (http:// www.itu.int/internetofthings/).

VARIAN H. et SHAPIRO C. (1999), Économie de l'information: guide stratégique de l'économie des réseaux, De Boeck Université.

VON HIPPEL E. (2005), Democratising innovation, The MIT Press.

WANG F. (2006), « Domain names management and legal protection », International Journal of Information Management, 26, pp. 116-127.

WARUSFEL B. (2004), « La protection des bases de données en question: un autre débat sur la propriété intellectuelle européenne », Propriétés intellectuelles, $\mathrm{n}^{\circ} 13$, octobre, pp. 896906.

WARUSFEL B. (2003), « La brevetabilité des méthodes commerciales devant les tribunaux français », Propriétés Intellectuelles, avril, $\mathrm{n}^{\circ} 7$.

\section{Lexique des acronymes}

ADPIC: Accord sur les aspects des droits de propriété intellectuelle qui touchent au commerce

DNS : Domain Names System

DPI: $\quad$ Droits de Propriété Intellectuelle

IP: $\quad$ Internet Protocol

ICANN : Internet Corporation for Assigned Names and Numbers

MPEG2: Moving Picture Expert Group

NSF: National Science Foundation

NSI: Network Solution Inc.

OEB : Office Européen des Brevets

OMC: Organisation Mondiale du Commerce

OMPI: $\quad$ Organisation Mondiale de la Propriété Intellectuelle

PI: $\quad$ Propriété Intellectuelle

TIC: Technologies de l'Information et de la Communication

TLD: Top Level Domains

cc TLD: country code Top Level Domain

g TLD: generic Top Level Domain

UDRP: Uniform Domain Name Dispute Resolution Policy

USPTO: US Patent and Trademark Office 